\title{
UTILIZAÇÃO DE COTS EM NANO SATÉLITES
}

\begin{abstract}
Junqueira, B. C. ${ }^{a}$; Manea, S. ${ }^{b}$
[a] Mestrando em Engenharia e Tecnologia Espaciais - Instituto Nacional de Pesquisas Espaciais (INPE) - Av. dos Astronautas, 1.758 - Jardim da Granja, São José dos Campos - SP, 12227-010. E-mail: bruno.junqueira@inpe.br [b] Doutor Tecnologista - Instituto Nacional de Pesquisas Espaciais (INPE) - Av. dos Astronautas, 1.758 - Jardim da Granja, São José dos Campos - SP, 12227-010. E-mail: silvio.manea@inpe.br

Resumo: $O$ desenvolvimento de nano satélites vem crescendo significativamente há alguns anos.Universidades e empresas têm cada vez mais interesse nesses sistemas espaciais, devido principalmente ao baixo custo e curto tempo de desenvolvimento. Os componentes comerciais (COTS - Commercial-Off-The-Shelf) são a base do desenvolvimento desses satélites; contudo, os componentes utilizados possuem baixa resistência à radiação, o que diminui sua confiabilidade no ambiente espacial. Neste artigo serão abordadas estratégias de utilização de transistores COTS, sendo similar para os demais componentes semicondutores, integrados ao sistema de forma a resistirem por mais tempo expostos à radiação. Com este intuito, um estudo dos efeitos da radiação para cada tipo de componente se faz necessário, analisando o quanto de radiação normalmente é observado que o componente resista de acordo com sua missão e quais são os efeitos gerados, para assim observar quais funções são melhores desempenhadas pelo componente, juntamente com as estratégias para a mitigação dos danos gerados. Desta forma, é possivel aumentar a vida útil do sistema.
\end{abstract}

Palavras-chave: COTS, Nano Satélites, Radiação, Engenharia de Sistemas.

\section{INTRODUÇÃO}

Os nano satélites possuem um peso entre 1 e $10 \mathrm{Kg}$, por este motivo seu custo de lançamento é significativamente menor que dos satélites convencionais, podendo ser através de veículos lançadores menores e mais baratos, além de poderem ser lançados em conjunto. Por esta acessibilidade e o interesse crescente na utilização de sistemas que requerem o segmento espacial, os nano satélites estão cada vez mais ganhando visibilidade.

Considerando este interesse nos custos, os nanosatélites são baseados normalmente em componentes COTS (Commercial-Off-The-Shelf), devido a sua disponibilidade e custos reduzidos. Os componentes de categoria espacial possuem uma série de restrições, pela sua criticidade, impossibilitando na maior parte das vezes sua aquisição. Transistores são um importante fator para o desenvolvimento desses satélites, e geralmente a radiação é uma questão crucial para estes componentes.

No presente artigo será observado uma estratégia de aplicação de componentes COTS para possibilitar um maior tempo de vida ao satélite, de modo a não acrescentar muitos custos.

$\mathrm{O}$ ambiente espacial possui uma série de características que o componente deve suportar, as principais são radiação, degaseificação, vácuo, microgravidade, oxigênio atômico, alta e baixa temperatura com variações rápidas, além da vibração durante o lançamento. Dentre estas questões, a radiação é um fator crítico porque os componentes COTS não são projetados para serem resistentes à radiação.

Para utilização de componentes COTS em projetos espaciais, ocorre a verificação dos componentes por meio de testes, o que gera custo elevado e tempo considerável. Para nano satélites estes testes não foram previstos no escopo original de seu desenvolvimento e desta forma, esta solução não tem sido aplicada.

\section{DISCUSSÃO}

Historicamente o surgimento da utilização de componentes COTS se deu pela mudança de políticas de consumo, marcado no século anterior pela influência da necessidade dos estados na aquisição e uso dos 
dispositivos e componentes eletrônicos. No fim do século, a indústria eletrônica passou a ter um mercado mais amplo, principalmente ao consumo individual, provocando grandes mudanças na indústria eletroeletrônica (Zednícek, 2016).

Como consequência, começou-se a declinar a influência da indústria espacial sobre os fabricantes globais, mas os SCM (Specialist Component Manufacturers - Fabricantes de Componentes Especializados) tornaram-se de importância crítica, trazendo inovações, flexibilidade e soluções especiais (Zednícek, 2016).

Porém, mesmo com os SCMs, pode-se haver a necessidade da utilização de COTS por parte direta da indústria aeroespacial, sendo os principais fatores a disponibilidade para aquisição e a evolução tecnológica muito acelerada. Normalmente, para o componente ser classificado como de uso espacial demora-se um tempo, e o desempenho é a principal característica desse avanço, para as missões mais disruptivas, estar no ápice da tecnologia é um fator crítico (Pignol, 2010).

Para componentes mais simples, como o caso de transistores, o fator mais crítico está na disponibilidade. Muitas vezes os componentes Hi-Rel (Alta Confiabilidade) / Rad-Tol (Tolerante à Radiação) I Rad-Hard (Endurecido à Radiação) não estão disponíveis, sendo necessário tempo de espera, podendo atrasar o andamento do projeto espacial.

No caso de nano satélites não fabricados pela indústria aeroespacial, os componentes COTS vêm para permitir que ocorra esse desenvolvimento por parte das empresas e universidades, de forma mais acessível. Normalmente esses componentes não possuem grande confiabilidade e pouco tempo de vida útil, porém são significativamente mais econômicos e de desenvolvimento rápido, permitindo profissionais e estudantes sem muitos recursos desenvolverem sistemas espaciais (Berk, 2013).

Devido à complexidade do sistema espacial, mesmo para nano satélites, o projeto deve ser abordado de forma sistêmica. Neste contexto, a engenharia de sistemas é a arte e a ciência de desenvolver um sistema operacional capaz de atender aos requisitos dentro de restrições impostas (NASA, 2016).

O processo de projetar consiste em muitos elementos interdependentes, com diversas interações, em vários estágios. Conforme estipulado pela engenharia de sistemas, o projeto se inicia em alto nível de abstração, de acordo com seu avanço, os níveis de abstração descem, porém podem ocorrer inconformidades e mudanças. Ao integrar o sistema, ocorre o processo inverso de abstração, onde são verificadas as conformidades, e por fim em operação é validado (NASA, 2016) (Berk, 2013). A Figura 1 ilustra o desenvolvimento do ciclo de vida de um sistema espacial, conforme ECSS-M-ST-10C, 2009.

Figura 1. Ciclo de vida do sistema espacial, conforme diretrizes do padrão ECSS-M-ST-10C.

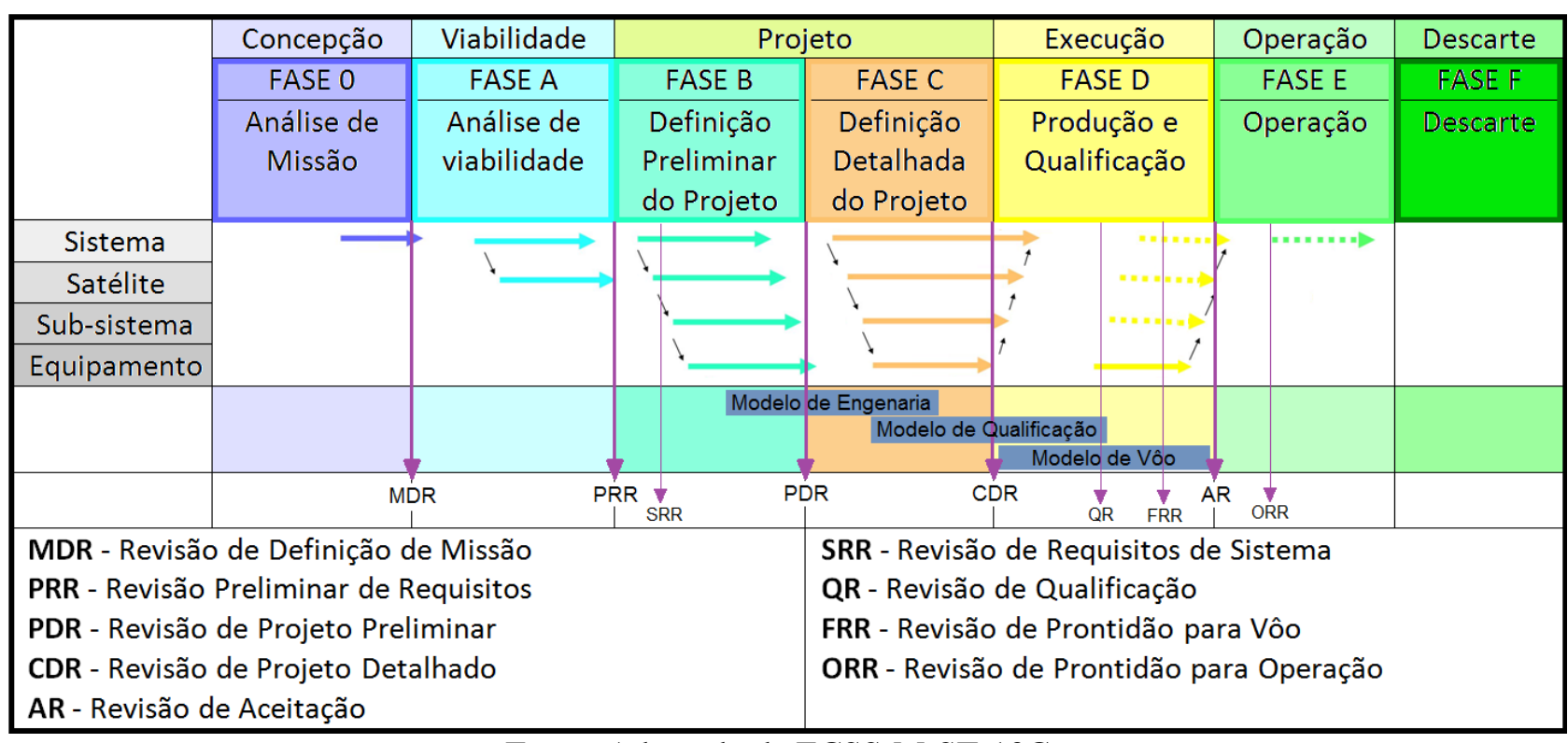


Os elementos-chave do planejamento e implementação do projeto e a identificação dos requisitos e produtos de nível superior, em conjunto, proporcionam um planejamento de projetos coerente e integrado (ECSS-M-ST-10C). Desta forma, é de suma importância o desenvolvimento do projeto em agregação construtiva e compatível do sistema, com a devida sequência de desenvolvimento.

No caso dos nano satélites esse processo de desenvolvimento possui algumas reduções em relação aos projetos convencionais, porém a base de desenvolvimento é similar. Um CubeSat por exemplo, de acordo com o CubeSat 101, 2017, pode ser projetado, construído, testado e entregue em apenas 9 meses, mas normalmente leva de 18 a 24 meses para ser concluído. Quando o CubeSat estiver pronto para ser entregue, o tempo típico para o lançamento é de alguns meses a alguns anos, dependendo da disponibilidade de oportunidades de lançamento e flexibilidade dos requisitos orbitais estipulados para a missão.

\subsection{O ambiente espacial e a radiação}

A radiação ao interagir com o material do componente eletrônico provoca diferentes efeitos, dependendo do: tipo de radiação, taxa de interação, tipo do material, processo e estrutura do material (Manea, 2018).

No momento da interação a radiação perde energia na matéria, podendo ser de três denominações diferentes dependendo da interação e do tempo de exposição do componente; são eles: efeito ionizante de longo prazo ou dose acumulada (TID - Total Ionizing Dose), efeito não ionizante de longo prazo (DDD Displacement Damage Dose), efeito ionizante de curto prazo (SEE - Single Event Effect). Na Figura 2 é mostrado esquematicamente a divisão dos danos por radiação nos dispositivos eletrônicos.

Figura 2. Esquema dos danos por radiação nos dispositivos eletrônicos.

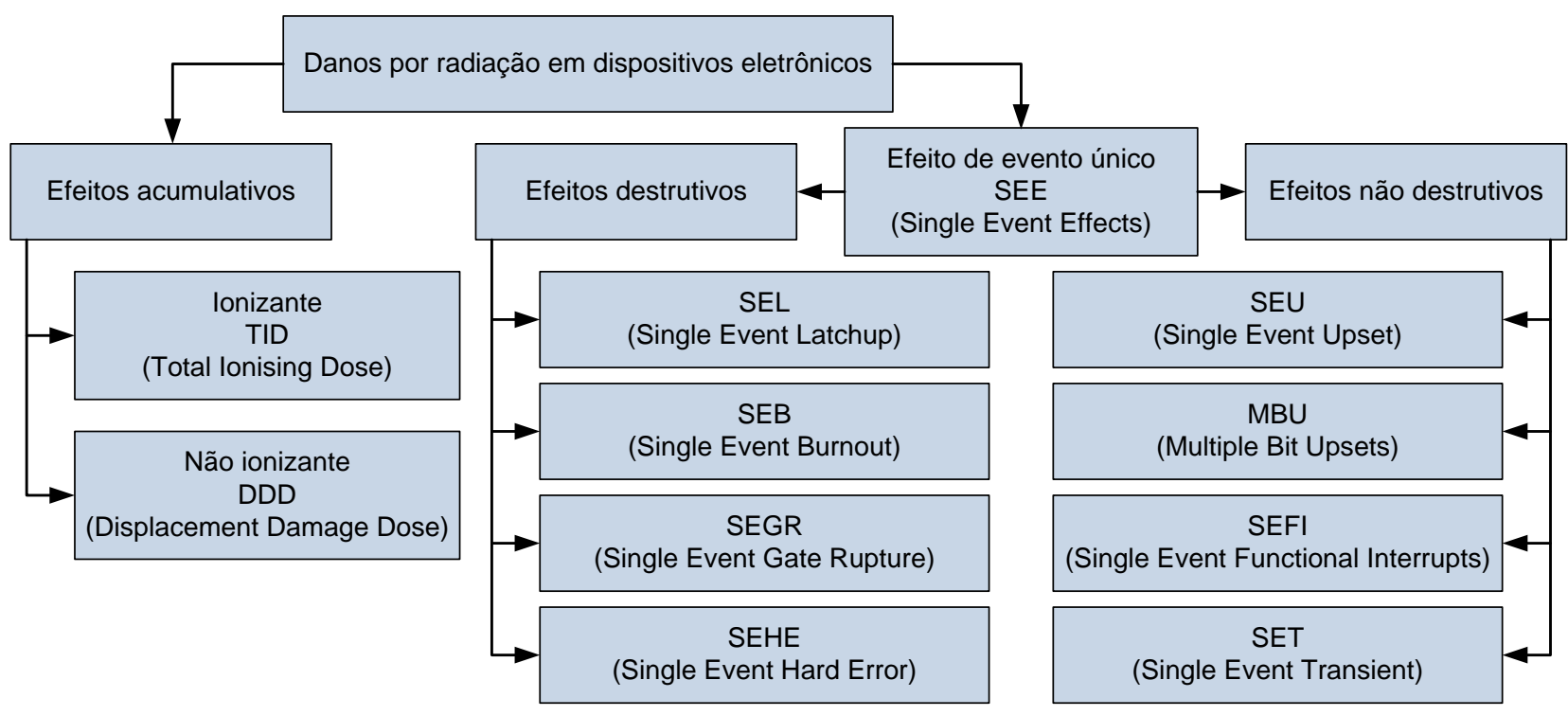

Fonte: Autor.

O TID se caracteriza pela formação de pares elétrons lacunas ao longo do tempo, onde os elétrons possuem maior mobilidade e atingem rapidamente a região condutora; já as lacunas se acumulam no óxido do dispositivo, principalmente na região não condutora de interface, perturbando a estrutura cristalina local que contém prótons ou hidrogênio ionizado, frequentemente liberando hidrogênio. Este processo muda a característica funcional do componente, dependendo de sua estrutura física e da qualidade do material e são provocados diferentes graus do efeito (Poizat, 2017). A Figura 3 ilustra este mecanismo. 


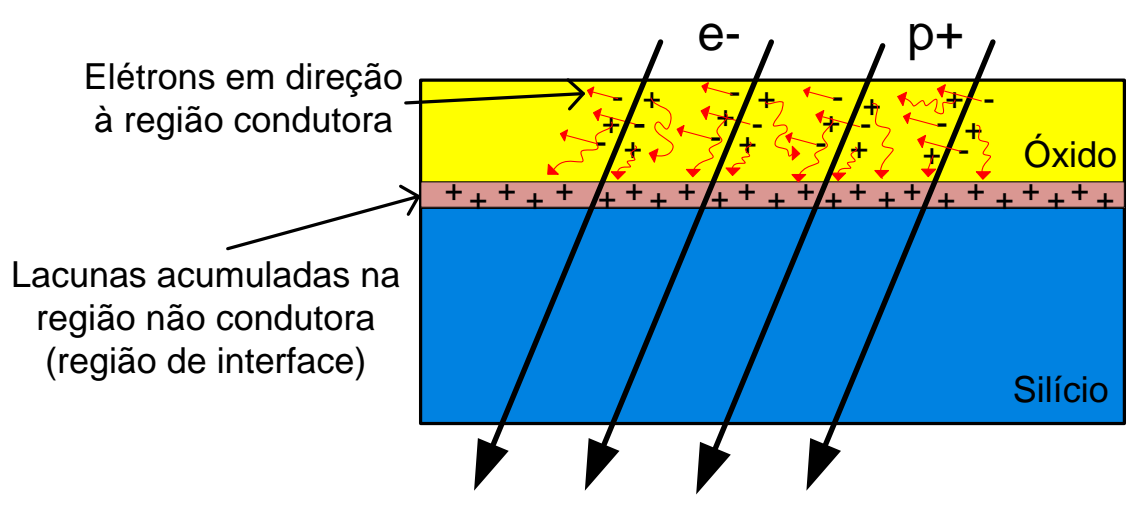

Fonte: Autor.

No DDD, a colisão entre as partículas incidentes e os átomos da rede faz com que a estrutura do material seja retirada da posição normal; quanto maior a energia, mais desta energia é transferida para o átomo de recuo que novamente pode continuar a criar átomos adicionais de recuo, portanto gerando uma cascata de defeitos na estrutura (Poivey, 2017). No decorrer do tempo, o componente vai se degradando assim como no TID. A Figura 4 ilustra o DDD.

Figura 4. Esquema representativo da Dose de Dano de Deslocamento (DDD).

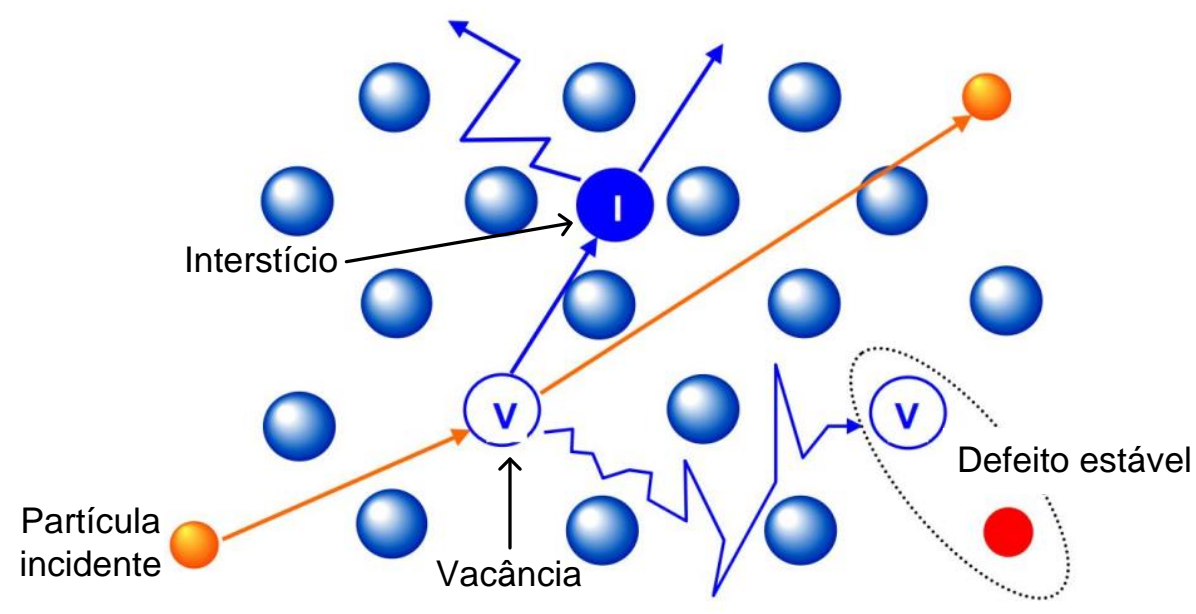

Retirado de: Poivey, 2017.

O SEE é resultado de uma única partícula cruzando uma área sensível do dispositivo ou circuito; dependendo da partícula e de sua energia, ocorre a deposição direta de energia pela partícula, o que normalmente ocorre com íons pesados, gerando uma linha de ionização no percurso do íon; ou para o caso de partículas mais leves, ocorre um efeito de ionização em cascata (Polo, 2017). A Figura 5 ilustra o SEE. 
Figura 5. Esquema representativo do Efeito de Evento Único (SEE).

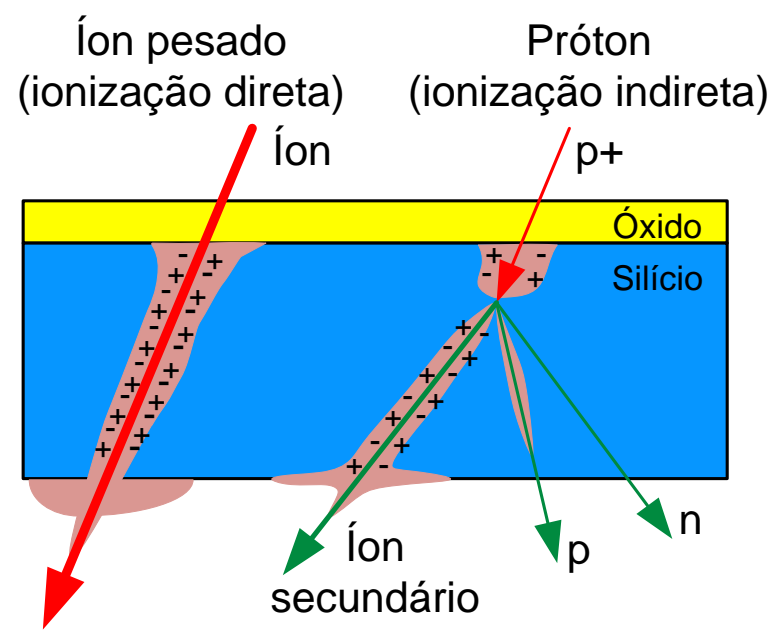

Fonte: Autor.

O evento pode ser destrutivo ou não destrutivo, dependendo do componente e da região atingida, gerando diversos efeitos. Nos eventos destrutivos ocorrem danos permanentes; já nos eventos não destrutivos não há dano físico, apenas perda de informações. De acordo com a norma ECSS-E-ST-10-12C, 2008 e MANEA, 2018, são descritos abaixo os principais efeitos do SEE.

Destrutivos:

- SEL (Single Event Latchup - Travamento por evento único) - Condição potencialmente destrutiva através de correntes parasitas, as quais podem ultrapassar o valor máximo suportado pelo componente.

- SEB (Single Event Burnout - "Queima" por evento único) - Alta corrente localizada no corpo do dispositivo, ativa o transistor bipolar parasítico, criando um caminho contínuo entre dreno e fonte. Sempre são destrutivos e os dispositivos com baixa dopagem são mais suscetíveis.

- SEGR (Single Event Gate Rupture - Ruptura de porta por evento único) - A partícula incidente do evento único atinge uma região de alto campo elétrico, formando um caminho condutor que resulta em correntes de fuga na porta e no dreno que excedem a corrente de vazamento normal.

- SEHE (Single Event Hard Error - Erro severo por evento único) - Relaciona-se à incapacidade de mudança de estado em células individuais, chamado de "bit preso" na memória.

Não destrutivos:

- SEU (Single Event Upsets - Perturbação por evento único) - Mudança de estado lógico, onde a partícula energizada colide com o nodo sensível de um circuito, tal como uma célula de memória e registradores.

- MBU (Multiple Bit Upsets - Perturbação de bit múltiplo) - Similar ao SEU, porém em mais de um bit.

- SEFI (Single Event Functional Interrupts - Interrupção funcional por evento único) Interrupção causada pela perturbação causada pela partícula ionizante, que leva a uma não funcionalidade temporária (ou interrupção da operação normal) do dispositivo afetado.

- SET (Single Event Transients - Transiente por evento único) - Efeito que gera um sinal espúrio no semicondutor e que pode se propagar e ser capturado como um valor lógico errado. 
Cada mecanismo de radiação possui suas principais fontes características, o que depende do tipo de partícula e da sua energia. A Tabela 1 faz esta relação.

Tabela 1. Efeitos da radiação relacionados as suas fontes.

\begin{tabular}{|l|l|}
\hline Mecanismo & \multicolumn{1}{c|}{ Fonte } \\
\hline TID & Prótons presos, elétrons presos, prótons solares. \\
\hline DDD & Prótons presos, elétrons presos, prótons solares, nêutrons. \\
\hline SEE & Íons pesados de raios cósmicos, prótons e íons pesados solares, prótons presos, nêutrons. \\
\hline
\end{tabular}

Modificado de: Manea, 2018.

As regiões orbitais possuem características de radiação diferentes, a região mais próxima da terra, nas órbitas mais baixas, possuem menores índices de radiação, porém logo acima delas, ainda na órbita LEO (Low Earth Orbit) há o cinturão interno de Van Allen, região com grande concentração de prótons capturados pelo campo magnético terrestre. Na região do Brasil e Oceano Atlântico Sul há uma anomalia em que o cinturão se aproxima mais da Terra.

Acima do cinturão interno há uma região sem grande acúmulo de partículas e, em seguida o cinturão externo com elétrons capturados. A Figura 6 ilustra a formação do cinturão de Van Allen. Além das partículas presas no cinturão há as partículas provenientes do Sol e do espaço profundo que são desviadas ou vão perdendo sua energia ao longo da trajetória até a Terra, pelo campo magnético terrestre.

Figura 6. Cinturão de Van Allen.

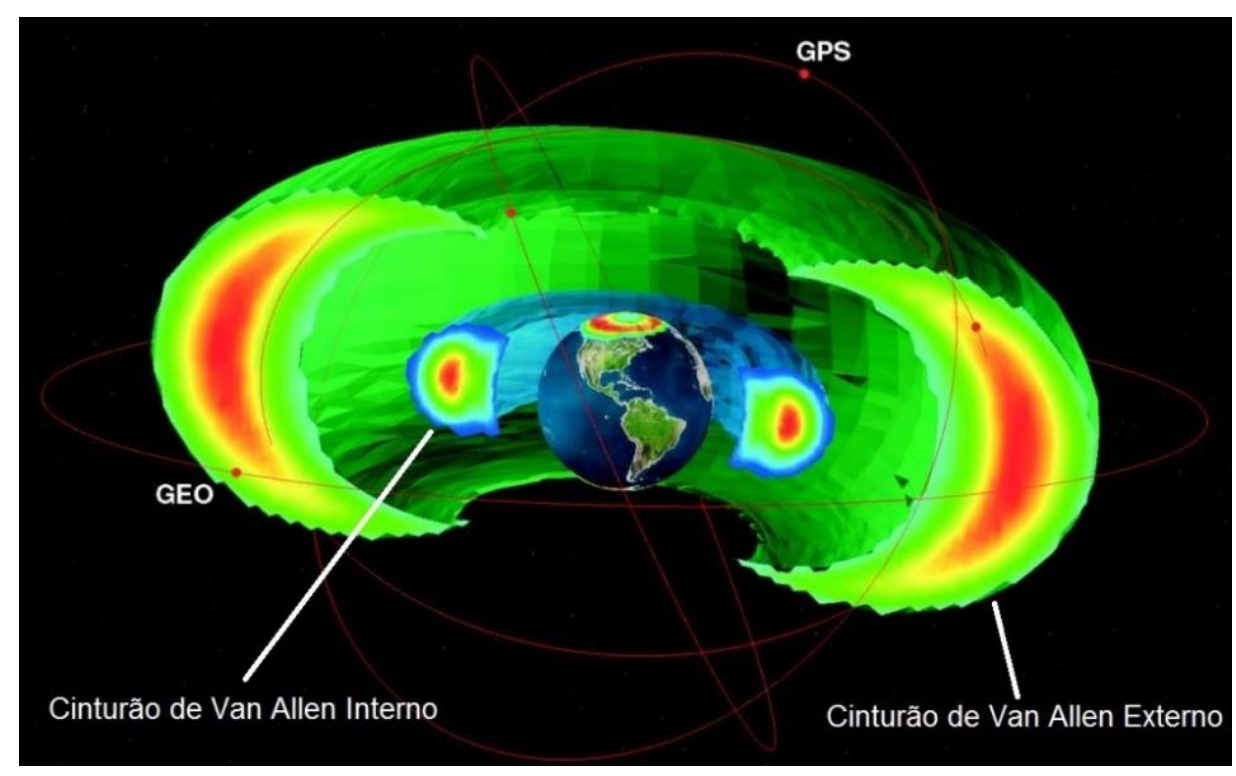

Retirado de: Manea, 2018.

Dependendo da região na qual o satélite irá orbitar, há maior incidência de determinadas partículas com energias específicas; podendo ser previstos quais os mecanismos mais frequentes de acordo com o componente e o tempo esperado para a missão (Manea, 2018).

No caso dos nano satélites, as órbitas mais comuns são órbitas baixas, na qual se tem a menor incidência de radiação. No caso dos satélites que devem operar na região do Brasil, a Anomalia do Atlântico Sul é uma dificuldade, pelo fato do satélite não poder se aproveitar de artifícios, como o desligamento do sistema, para se proteger dos prótons acumulados nesta região (Manea, 2018). 


\subsection{Efeitos da radiação nos componentes}

Neste trabalho o estudo se concentrará em componentes MOS e BJTs, analisando seus mecanismos com a exposição de radiação. A Figura 7 ilustra estes componentes.

Figura 7. Componentes MOS (MOSFET canal N) e BJT (NPN).

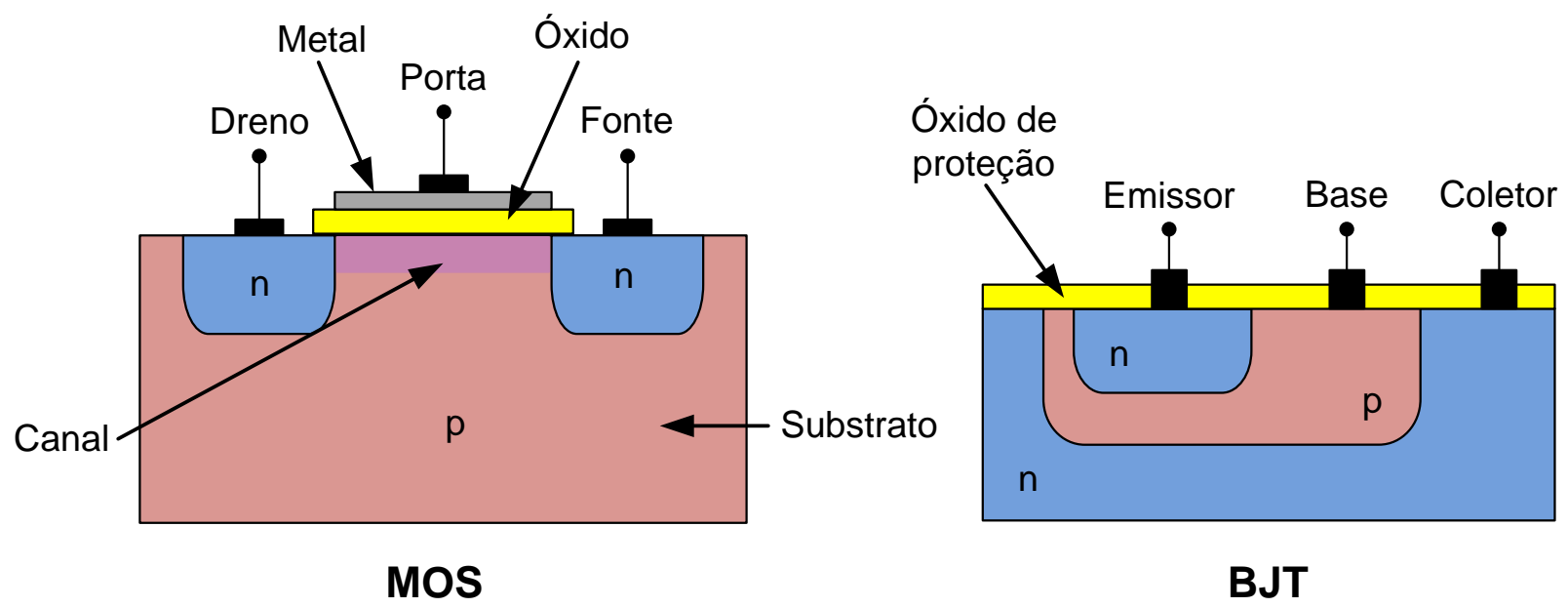

Fonte: Autor.

No NMOS, aplicando-se uma tensão adequada na porta cria-se um canal de condução entre a fonte e o dreno, ligando o dispositivo. Devido a dose de radiação ionizante, cargas presas no óxido de entrada causam deslocamento da tensão limiar necessária para fazer esse acionamento. Se o deslocamento for significativo o suficiente, o transistor não pode ser desligado mesmo com a tensão de porta igual a zero. No caso do PMOS ocorre o mesmo processo, porém ao contrário, o transistor não pode ser ligado mesmo com a tensão de porta máxima (Poizat, 2017) (Löchner, 2011).

A tecnologia MOS é amplamente imune ao mecanismo DDD, devido a ocorrer somente em uma região muito pequena, o que não afeta o componente (Löchner, 2011). Para o SEE são observados SEL, SEB, SEGR, SEHE, SEU, MBU, SEFI e SET (ECSS-E-ST-10-12C, 2008). Dependendo da tecnologia, família e função do componente este estará sujeito a diferentes efeitos do SEE.

Já para os transistores bipolares (BJTs), o TID ocorre da mesma maneira que nos transistores MOS, porém os BJTs possuem a camada de óxido de proteção mais espessa, o que gera maior concentração de armadilha de carga e estados de interface. Provocando aumento da corrente de base e por consequência degradação do ganho, além das fugas de carga; são mais afetados por baixa taxa de dose (Poizat, 2017) (Löchner, 2011).

Assim como ocorre no TID, o DDD provoca degradação de ganho e corrente de fuga em transistores bipolares, devido a degradação da estrutura cristalina do material. Os transistores bipolares PNP são mais afetados por este mecanismo (Löchner, 2011). Para o SEE em BJTs é observado SEB, SET e SEU (ECSS-EST-10-12C, 2008).

\section{RESULTADOS}

O componente é integrado ao sistema de forma que atenda aos requisitos de acordo com as circunstâncias e cenários aplicáveis ao longo de sua vida útil. Para este trabalho foi observado a questão da radiação, porém os demais parâmetros devem ser considerados.

Pela análise dos efeitos da radiação no tópico anterior, observa-se que os componentes possuem tolerância a alguns mecanismos, mas são mais frágeis a outros. Neste contexto, deve-se observar a órbita e o 
tempo de missão pretendidos para o sistema, para se estimar a radiação predominante e sua fluência. A partir desses dados formular as estratégias de utilização dos componentes.

Cada mecanismo possui algumas características e cada categoria de componente reage de uma forma com a radiação incidente; por esta razão, as mitigações devem ser desenvolvidas para o sistema com critérios bem definidos.

No caso de SEE colocar blindagem, por exemplo, não representa uma mitigação considerando que a blindagem para proteger de altas energias deveria ser muito espessa, inviabilizando o projeto. No caso de TID e DDD, colocar redundâncias não é relevante para estes mecanismos, devido ao fato da radiação que provoca esses efeitos está sendo depositada ao longo do tempo de forma equivalente no sistema.

Já para os componentes, a radiação incidente pode provocar diferentes efeitos. Para o caso dos BJTs, com a degradação por TID, possuem maior resistência para o chaveamento; já para o ganho, ocorre uma queda dos parâmetros de fabricação. Então, dependendo da função, há diferentes graus de degradação.

Outro fator importante a ser considerado é a densidade, onde quanto menor a densidade do dispositivo, mais resistente à TID, porém são mais suscetíveis a SEE.

A Tabela 2 faz uma relação entre os componentes BJTs e MOS com os mecanismos de radiação e as soluções aplicáveis para cada categoria de componentes.

Tabela 2. Efeitos da radiação em componentes BJTs e MOS e as soluções aplicáveis.

\begin{tabular}{|c|c|c|c|}
\hline $\begin{array}{l}\text { Categoria do } \\
\text { componente }\end{array}$ & Mecanismo & Efeitos & Soluções \\
\hline \multirow[t]{5}{*}{$\mathrm{BJT}$} & TID & $\begin{array}{l}\text { Degradação do fator de ganho, } \\
\text { principalmente para condições } \\
\text { de baixa corrente, PNP mais } \\
\text { sensível. Baixa dose de } \\
\text { radiação provoca efeitos } \\
\text { piores. }\end{array}$ & $\begin{array}{l}\text { Utilizar transistor com o ganho um } \\
\text { pouco acima do estipulado, para } \\
\text { melhorar a margem ao longo da } \\
\text { degradação do componente. Colocar uma } \\
\text { blindagem para diminuir a incidência de } \\
\text { radiação de baixa energia. }\end{array}$ \\
\hline & $\overline{\mathrm{DDD}}$ & $\begin{array}{l}\text { Degradação do fator de ganho, } \\
\text { principalmente para condições } \\
\text { de baixa corrente, PNP mais } \\
\text { sensível. }\end{array}$ & $\begin{array}{l}\text { Utilizar transistor com o ganho um } \\
\text { pouco acima. Blindagem. }\end{array}$ \\
\hline & \multirow[t]{3}{*}{ SEE } & SEB & $\begin{array}{l}\text { Redundância. Detector de aumento } \\
\text { súbito de corrente para efetuar o } \\
\text { desligamento do circuito. }\end{array}$ \\
\hline & & SET & Sistemas de detecção e correção de erros. \\
\hline & & SEU & Sistemas de detecção e correção de erros. \\
\hline \multirow[t]{6}{*}{ MOS } & TID & $\begin{array}{l}\text { Mudança de tensão de } \\
\text { acionamento ou desligamento }\end{array}$ & $\begin{array}{l}\text { Blindagem. NMOS com a degradação } \\
\text { fica com vazamento, e PMOS fica com } \\
\text { bloqueio; implementar de modo a } \\
\text { considerar esses fatos. }\end{array}$ \\
\hline & DDD & Amplamente imune & - \\
\hline & \multirow[t]{4}{*}{ SEE } & SEL & $\begin{array}{l}\text { Detector e recuperação por ciclo de } \\
\text { energia. }\end{array}$ \\
\hline & & SEB & $\begin{array}{l}\text { Redundância. Detector de aumento } \\
\text { súbito de corrente e desligamento }\end{array}$ \\
\hline & & SEGR & $\begin{array}{l}\text { Redundância. Isolamento do circuito } \\
\text { corrompido. }\end{array}$ \\
\hline & & SET & Sistemas de detecção e correção de erros \\
\hline
\end{tabular}




\begin{tabular}{|l|l|l|}
\hline & SEFI & Recuperado por reset ou ciclo de energia \\
\cline { 3 - 4 } & SEU & Sistemas de detecção e correção de erros \\
\cline { 2 - 3 } & SEHE & $\begin{array}{l}\text { Ciclismo de energia. Sistemas de } \\
\text { detecção e correção de erros. }\end{array}$ \\
\hline
\end{tabular}

Fonte: Autor

As mitigações representam uma boa solução no âmbito de componente e de sistema, porém devem ser muito bem dosadas, pois cada mitigação implementada aumenta a complexidade do sistema, o que pode resultar em mais pontos de falha, aumento de peso, volume, consumo de energia e dificuldade de desenvolvimento do satélite.

Outro fator importante é para qual aplicação o dispositivo foi desenvolvido, o que pode representar uma informação sobre o quanto da qualidade e de testes observados o dispositivo possui. As principais aplicações observadas são: militar, automotivo, médico, industrial, comercial. Dependendo da aplicação para a qual o componente foi desenvolvido, uma série de requisitos são cobertas por estes dispositivos, porém a radiação não é uma delas.

A partir das decisões citadas, observando a missão e escolhendo o componente para exercer uma determinada função com as mitigações de modo dosado, é possível aumentar a vida útil e confiabilidade do sistema sem agregar muitos custos.

\section{ANÁLISE}

Na pesquisa de componentes críticos para sistemas espaciais desenvolvida no INPE, foi caracterizado em relação a TID o transistor Bipolar NPN 2N2222A a uma dose acumulada de $150 \mathrm{Krads}(\mathrm{Si})$ numa taxa de dose de $1 \mathrm{krad} / \mathrm{h}$. O objetivo deste ensaio foi investigar e avaliar a tolerância aos efeitos da dose total de radiação ionizante no qual o componente será submetido no ambiente espacial e para diagnosticar lotes de componentes que possuam defeitos de fabricação ou que tenham sido causados por outros fatores que se manifestarão no início da vida útil do dispositivo. A avaliação foi realizada de acordo com as normas internacionais de radiação. Um conjunto completo de medições elétricas foram realizadas, juntamente com a representação gráfica dos parâmetros medidos com respeito à dose total irradiada em todas as amostras (Junqueira, 2018).

Através dos testes verificou-se que os transistores para a função de amplificadores não permaneceram de acordo com as especificações do datasheet, o que demonstra que os mesmos não são tolerantes aos níveis de radiação aplicados. Porém, para a função de chaveamento, a variação dos parâmetros não interferiu significativamente na operação do componente, demonstrando que este atende ao uso como chave no sistema eletrônico do satélite (Junqueira, 2018).

Este teste demonstra que componentes que não são resistentes à radiação, para certas funções podem atender muito bem os parâmetros estipulados. Servindo não somente para transistores, mas para qualquer componente semicondutor.

Para o caso dos nano satélites onde a concepção é de menor tempo e custo de desenvolvimento, testes de radiação não são viáveis, mas através de uma análise é possível aplicar o componente da forma que mais o beneficie aumentando assim a vida útil do sistema.

\section{CONCLUSÕES}

O presente trabalho constituiu-se na análise da radiação em transistores MOS e BJTs COTS para aplicação em nano satélites, observando estratégias para o uso desses componentes no ambiente espacial. Este padrão também aplica-se aos demais componentes semicondutores.

Muitas vezes não é dada a devida atenção aos efeitos da radiação incidente nos componentes eletrônicos implementados nos nano satélites, o que pode comprometer o sistema e a missão do satélite. A escolha e integração adequadas do componente, analisando os parâmetros afetados e a forma de falha, são de suma 
importância para melhor implementação dos COTS ao sistema, o que pode aumentar consideravelmente a vida útil do satélite.

Dosar adequadamente na escolha e implementação dos componentes é a chave para o sucesso da missão, onde esta escolha poderá ocasionar em perdas e acréscimos ao sistema. Analisar as menores perdas e maiores acréscimos de acordo com o benefício da missão não é uma tarefa fácil, mas deve ser desenvolvida para o benefício da missão.

\section{AGRADECIMENTOS}

Ao Instituto Nacional de Pesquisas Espaciais (INPE) pelo ambiente de pesquisa, profissionais e corpo docente, e à CAPES pelo apoio financeiro.

\section{REFERÊNCIAS}

Berk, J., Straub, J., Whalen, D., 2013. The open prototype for educational NanoSats: Fixing the other side of the small satellite cost equation. 978-1-4673-1813-6/13/\$31.00 (02013 IEEE.

CubeSat 101, Basic Concepts and Processes for First-Time CubeSat Developers. NASA CubeSat Launch Initiative For Public Release - Revision Dated October 2017.

EUROPEAN COOPERATION FOR SPACE STANDARDIZATION ( ECSS). ECSS-E-ST-10-12CMethods for the calculation of radiation received and its effects, and a policy for design margins. Noordwijk, The Netherlands, 2008.

EUROPEAN COOPERATION FOR SPACE STANDARDIZATION ( ECSS). ECSS-MST-10C- Space project management, project planning and implementation. Noordwijk, The Netherlands, 2009.

Junqueira, Bruno C.; Manea, Silvio; Vaz, Rafael G.; Gonçalez, Odair L.Teste em Componente Crítico de uso Espacial: Ensaio e Dose Ionizante Total, (TID - Total Ionizing Dose) em Transistores 2N2222A. INPE, $9^{\circ}$ Workshop em Engenharia e Tecnologia Espaciais, 2018.

Löchner, S., 2011. Radiation Damages to Electronic Components. Strahlungsschädigungen an elektronischen Bauteilen.

Manea, S., 2018. Introduction to the Space Radiation Effects on Satellites, CSE-342-4. INPE. Class notes

NASA Systems Engineering Handbook - NASA SP-2016-6105 Rev2. Disponivel em: <https://www.nasa.gov/sites/default/files/atoms/files/nasa_systems_engineering_handbook_0.pdf> Acesso: 5 de abril de 2019

Pignol, M., 2010. COTS-based Applications in Space Avionics. CNES, Toulouse, France.

Poivey, C., 2017. TNID Total Non Ionizing Dose or DD Displacement Damage.ESA - CERN - SCC Workshop.

Poizat, M., 2017. Radiation Environment and its Effects in EEE Components and Hardness Assurance for Space Applications. TID Total Ionizing Dose.European Space Agency.

Polo, C. B., 2017. Radiation Environment and its Effects in EEE Components and Hardness Assurance for Space Applications. SEE Single Event Effects. ESA-CERN-SCC Workshop, CERN

Zednícek, T., 2016. Commercial versus COTS + versus Qualified Passive Components in Space Applications. SA/ESTEC, Noordwijk, The Netherlands. 